\title{
Condutividade elétrica e teor de água inicial das sementes de soja ${ }^{(1)}$
}

\begin{abstract}
Roberval Daiton Vieira ${ }^{(2)}$, Adilson Luís Penario|(3), Dilermando Perecin ${ }^{(4)}$ e Maristela Panobianco ${ }^{(5)}$
Resumo - O teor de água das sementes é fator de extrema importância na padronização do método do teste de condutividade elétrica, visto que influencia significativamente os resultados. O objetivo deste trabalho foi estudar o efeito do teor de água inicial sobre os resultados da condutividade elétrica das sementes de soja, e determinar uma equação capaz de corrigir a sua influência. Foram utilizadas seis cultivares de soja, em dois anos agrícolas, cada uma representada por lotes com três níveis de vigor. Foi ajustado o teor de água das sementes de cada lote para 7, 9,11,13,15 e 17\%, antes da condução do teste de condutividade elétrica; a equação de correção para ajuste dos dados foi determinada com base em regressões, com os resultados dos dois anos agrícolas, cultivares e níveis de vigor. O teor de água inicial das sementes de soja pode influenciar a interpretação dos resultados da condutividade elétrica e a equação de correção é eficiente para eliminar o efeito do teor de água sobre os resultados do teste.
\end{abstract}

Termos para indexação: Glycine max, vigor da semente, teste de sementes, equação de correção.

\section{Electrical conductivity and initial water content of soybean seeds}

\begin{abstract}
The initial seed water content can influence significantly the electrical conductivity results, and can make difficult the test standardization. The present work was carried out in order to study the effect of initial seed water content on the electrical conductivity results and to determine an equation to correct this influence. Six soybean cultivars were used, for two years, each cultivar having lots with high, medium and low seed vigor. Seed water content of each lot was adjusted to 7, 9, 11,13,15 and 17\% before electrical conductivity test was run. The equation to correct the electrical conductivity values was calculated based on regression, including the two years data. The initial seed water content proved to be of high influence on the electrical conductivity interpretation and the equation is efficient to eliminate the effect of initial seed water content on the electrical conductivity data.
\end{abstract}

Index terms: Glycine max, seed vigor, seed testing, adjustment equation.

\section{Introdução}

Os testes de vigor têm sido instrumentos de uso cada vez mais rotineiro pela indústria de sementes para determinação da sua qualidade fisiológica.

(1) Aceito para publicação em 19 de dezembro de 2001 .

(2) Universidade Estadual Paulista (Unesp), Faculdade de Ciências Agrárias e Veterinárias (FCAV), Dep. de Produção Vegetal, Campus de Jaboticabal, Via de Acesso Prof. Paulo Donato Castellane, s/no, CEP 14884-900 Jaboticabal, SP. Bolsista do CNPq. E-mail: rdvieira@fcav.unesp.br

(3) Monsanto do Brasil, Rua Eduardo de Oliveira, 940, CEP 38400-174 Uberlândia, MG. E-mail: adilson.penariol@monsanto.com

(4) Unesp, FCAV, Dep. de Ciências Exatas. Bolsista do CNPq. E-mail: perecin@fcav.unesp.br

(5) Av. General Osório, 120, ap. 51, CEP 14870-000 Jaboticabal, SP. E-mail: mapanobianco@uol.com.br
As empresas produtoras e as instituições oficiais têm incluído esses testes em programas internos de controle de qualidade ou para a garantia da qualidade das sementes destinadas à comercialização (Marcos Filho, 1999).

Freqüentemente, lotes de sementes que apresentam germinação semelhante exibem comportamentos distintos no campo ou armazenamento. Essas diferenças podem ser explicadas pelo fato de que as primeiras alterações nos processos bioquímicos associados à deterioração ocorrem, geralmente, antes que sejam verificados declínios na capacidade germinativa (Delouche \& Baskin, 1973). Por este motivo, o uso de testes de vigor é de grande utilidade no monitoramento da qualidade das sementes, a partir da maturidade (Dias \& Marcos Filho, 1995).

Considerando-se que os testes de vigor fornecem índices mais sensíveis do potencial fisiológico, 
quando comparados ao teste de germinação (Association of Official Seed Analysts, 1983), qualquer evento que preceda a perda do poder germinativo pode servir como base para o desenvolvimento de testes de vigor. Entretanto, acredita-se que, quanto mais próximo da maturidade fisiológica (ou mais distante da perda do poder germinativo) estiver a variável avaliada, mais sensível deverá ser o teste. Como a degradação das membranas celulares se constitui, hipoteticamente, no primeiro evento do processo de deterioração (Delouche \& Baskin, 1973), os testes que avaliam a integridade das membranas seriam, teoricamente, os mais sensíveis para estimar o vigor.

Neste sentido, pode-se destacar o teste de condutividade elétrica, no qual a qualidade das sementes é avaliada indiretamente através da determinação da quantidade de lixiviados na solução de embebição das sementes. Os menores valores, correspondentes à menor liberação de exsudatos, indicam alto potencial fisiológico (maior vigor), revelando menor intensidade de desorganização dos sistemas membranais das células.

Os resultados da condutividade elétrica podem ser influenciados por vários fatores, como: presença de sementes danificadas fisicamente (Tao, 1978; Loeffler et al., 1988), tamanho da semente (Tao, 1978; Deswal \& Sheoran, 1993), genótipo de uma mesma espécie (Panobianco \& Vieira, 1996; Vieira et al., 1996, 1998; Panobianco et al., 1999), teor de água inicial das sementes (Association of Official Seed Analysts, 1983; Loeffler et al., 1988; Hampton et al., 1992; Carvalho, 1994), período de embebição (Loeffler et al., 1988; Wang et al., 1994) e temperatura de embebição (Murphy \& Noland, 1982; Gilvelberg et al., 1984).

Dentre os fatores citados, o teor de água das sementes por ocasião da realização da condutividade elétrica é de extrema importância na padronização do método do teste, bem como na obtenção de resultados uniformes entre laboratórios e dentro de um mesmo laboratório. Dependendo da espécie, da região de produção, da época de colheita, da eficiência da secagem, e do ambiente, pode ser encontrada uma amplitude de variação muito grande entre os valores (Hampton et al., 1994).

Em geral, tem-se verificado que teores de água muito baixos $(\leq 10 \%)$ ou muito altos $(\geq 17 \%)$ apresen- tam influência significativa nos resultados. Recomenda-se a uniformização dos teores de água dos lotes para uma faixa entre $10 \%$ e $17 \%$, antes da avaliação da condutividade elétrica (Association of Official Seed Analysts, 1983; Loeffler et al., 1988; Hampton et al., 1992). Efeito maior tem sido observado quando o teor de água das sementes é muito baixo $(\leq 10 \%)$, causando aumento significativo nos resultados do teste para várias espécies.

Uma alternativa para minimizar a influência do teor de água inicial das sementes, nos resultados do teste, é a determinação de um fator de correção, baseado em um teor de água padrão. Loeffler et al. (1988) sugeriram que os valores da condutividade elétrica determinados para sementes de soja deveriam ser corrigidos, com base na leitura realizada em sementes com teores de $13 \%$ de água; isto poderia ser feito utilizando-se um fator de correção calculado pela divisão do valor da condutividade elétrica obtida com o teor de água de $13 \%$ (padrão) pelo valor obtido nos outros teores. Assim, Carvalho (1994), utilizando um fator de correção para 13\% de água, obteve o mesmo comportamento de condutividade elétrica em lotes com teores variando de 7,0\% a 14,5\% de água. No entanto, este último autor salientou a necessidade da obtenção de diferentes fatores de correção em cada cultivar, o que dificulta a rotina dos laboratórios, uma vez que a renovação de cultivares é muito rápida.

O objetivo deste trabalho foi estudar o efeito do teor de água inicial sobre os resultados da condutividade elétrica de sementes de soja, e determinar uma equação capaz de corrigir sua influência.

\section{Material e Métodos}

O trabalho foi desenvolvido no Laboratório de Análise de Sementes do Departamento de Produção Vegetal da FCAV/Unesp, em Jaboticabal, SP, durante dois anos agrícolas.

Foram utilizadas seis cultivares de soja (Savana, Doko RC, IAC-17, IAC-15, IAS-5 e IAC-31 Foscarin), safras 94/95 e 95/96. Para cada cultivar selecionaram-se lotes com três níveis de vigor (alto, médio e baixo), com base nos testes de germinação, envelhecimento acelerado e condutividade elétrica.

Inicialmente, determinou-se o teor de água das sementes secadas em estufa a $105 \pm 3^{\circ} \mathrm{C}$, durante 24 horas, segun- 
do as instruções das Regras para Análise de Sementes (Brasil, 1992). Posteriormente, realizou-se o ajuste para seis níveis (7, 9, 11, 13, 15 e 17\% de água), da seguinte maneira: com base no teor de água inicial, calculou-se a massa que uma amostra de $300 \mathrm{~g}$ de sementes deveria atingir para apresentar os teores desejados; as amostras que necessitavam de redução da massa inicial foram colocadas em estufa com circulação de ar regulada à temperatura de $33^{\circ} \mathrm{C}$, em bandejas metálicas. As que necessitavam de aumento da massa, foram dispostas em rolos de papel (tipo Germitest), previamente umedecidos com quantidade de água equivalente a 2,5 vezes a massa do papel, mantidos a $20^{\circ} \mathrm{C}$, em câmara de germinação. Durante o umedecimento artificial, o teor de água das sementes foi monitorado, por pesagens sucessivas, até a obtenção dos valores desejados. As sementes foram, então, colocadas em vidros tampados, embalados em sacos de plástico, e mantidos em câmara fria $\left(10^{\circ} \mathrm{C}, 50-60 \%\right.$ de umidade relativa do ar) por sete dias, para que atingissem o equilíbrio higroscópico. Após este período, foi determinado o teor de água das sementes, pelo método da estufa a $105 \pm 3^{\circ} \mathrm{C}$ (Brasil, 1992), para confirmar a eficiência da técnica de umedecimento artificial utilizada.

$\mathrm{Na}$ avaliação da condutividade elétrica na solução de embebição das sementes, foram usadas quatro repetições de 50 sementes, pesadas com precisão de $0,01 \mathrm{~g}$ e colocadas para embeber em copos de plástico (capacidade de $200 \mathrm{~mL}$ ) contendo $75 \mathrm{~mL}$ de água deionizada, durante 24 horas, a $25^{\circ} \mathrm{C}$ (Association of Official Seed Analysts, 1983; Vieira \& Krzyzanowski, 1999). Em seguida, procedeu-se à leitura da condutividade em condutivímetro DIGIMED CD-21, sendo os resultados expressos em $\mu \mathrm{S} \mathrm{cm}^{-1} \mathrm{~g}^{-1}$ de semente.

Após a obtenção dos resultados de condutividade elétrica dos dois anos de estudo, foram determinados os fatores de correção com base no teor de água de $13 \%$, pois de acordo com a literatura, a estabilização dos resultados do teste ocorre quando as sementes de soja apresentam entre 11\% e 13\% de água (Loeffler et al., 1988; Carvalho, 1994). A determinação dos fatores de correção foi efetuada pela divisão do valor da condutividade elétrica obtida com o teor de $13 \%$ de água, pelo valor obtido em cada um dos outros teores.

Os fatores de correção, após serem calculados, foram analisados conjuntamente, através de regressão, utilizandose os resultados das duas safras agrícolas, obtendo-se a seguinte equação para ajuste dos dados de condutividade elétrica:

$\mathrm{CE}=[0,3227+0,05115(\mathrm{TA})] \times \mathrm{CE}_{\mathrm{o}}$

em que $\mathrm{CE}$ é a condutividade elétrica corrigida $\left(\mu \mathrm{S} \mathrm{cm}^{-1} \mathrm{~g}^{-1}\right)$; TA é o teor de água observado nas sementes $(\%) ; \mathrm{CE}_{\mathrm{o}}$ é a condutividade elétrica observa$\mathrm{da}\left(\mu \mathrm{S} \mathrm{cm}^{-1} \mathrm{~g}^{-1}\right)$.
O delineamento usado foi o inteiramente casualizado, em esquema fatorial $6 \times 3 \times 6$ (cultivares $\mathrm{x}$ níveis de vigor $\mathrm{x}$ teores de água), com quatro repetições. As médias das avaliações de condutividade elétrica foram comparadas pelo teste de Tukey, a 5\% de probabilidade.

\section{Resultados e Discussão}

Nas duas safras agrícolas, houve diferenças significativas entre os fatores, quando se consideram os resultados da condutividade elétrica de acordo com o nível de vigor e do teor de água inicial das sementes (Tabela 1). Nos três níveis de vigor, houve redução na condutividade elétrica à medida que o teor de água aumentou de $7 \%$ para $17 \%$; dentro do nível alto, ocorreram as menores variações nos resultados da condutividade elétrica, variações estas, que aumentaram com a redução do vigor.

As leituras de condutividade elétrica diminuíram quando se aumentou o teor de água das sementes, com tendência de estabilização entre as cultivares a partir de $13 \%$ de água (Tabela 2). Resultados semelhantes foram constatados por Loeffler et al.(1988) e Carvalho (1994). A cultivar Doko RC apresentou os menores valores, e IAC-17, os maiores. Analisando-se comparativamente com os resultados da condutividade elétrica corrigida, com base na equação proposta (Tabela 2), pode-se notar que todas as

Tabela 1. Condutividade elétrica observada $\mathrm{CE}_{\mathrm{o}}$ $\left(\mu \mathrm{S} \mathrm{cm}^{-1} \mathrm{~g}^{-1}\right)$ de sementes de soja, de acordo com o nível de vigor e o teor de água inicial. Safras 1994/95 e 1995/96(1).

\begin{tabular}{cccc}
\hline \multirow{2}{*}{$\begin{array}{c}\text { Teor de água } \\
(\%)\end{array}$} & Alto & Médio & Baixo \\
\cline { 2 - 4 } & & Safra 1994/95 \\
7 & $93 \mathrm{aC}$ & $118 \mathrm{aB}$ & $140 \mathrm{aA}$ \\
9 & $76 \mathrm{bC}$ & $100 \mathrm{bB}$ & $122 \mathrm{bA}$ \\
11 & $68 \mathrm{cC}$ & $87 \mathrm{cB}$ & $106 \mathrm{cA}$ \\
13 & $64 \mathrm{cC}$ & $78 \mathrm{~dB}$ & $96 \mathrm{dA}$ \\
15 & $56 \mathrm{dC}$ & $73 \mathrm{deB}$ & $89 \mathrm{eA}$ \\
17 & $57 \mathrm{dC}$ & $71 \mathrm{eB}$ & $87 \mathrm{eA}$ \\
\hline & & Safra $1995 / 96$ \\
7 & $86 \mathrm{aC}$ & $112 \mathrm{aB}$ & $134 \mathrm{aA}$ \\
9 & $72 \mathrm{bC}$ & $95 \mathrm{bB}$ & $109 \mathrm{bA}$ \\
11 & $65 \mathrm{cC}$ & $82 \mathrm{cB}$ & $97 \mathrm{cA}$ \\
13 & $57 \mathrm{dC}$ & $74 \mathrm{~dB}$ & $88 \mathrm{dA}$ \\
15 & $54 \mathrm{dC}$ & $67 \mathrm{eB}$ & $79 \mathrm{eA}$ \\
17 & $47 \mathrm{eC}$ & $60 \mathrm{fB}$ & $68 \mathrm{fA}$ \\
\hline
\end{tabular}

(1)Médias seguidas pela mesma letra, minúscula na coluna e maiúscula na linha, não diferem entre si pelo teste de Tukey, a 5\% de probabilidade; os coeficientes de variação relativos ao de vigor médio nas safras de $1994 / 95$ e $1995 / 96$ foram de $7,90 \%$ e $6,56 \%$, respectivamente. 
cultivares apresentaram redução na amplitude de variação entre os teores de água das sementes. A cultivar Savana mostrou uma amplitude de variação de $5 \mu \mathrm{S} \mathrm{cm}^{-1} \mathrm{~g}^{-1}$, bem menor do que a verificada antes da aplicação da equação de correção, ou seja, de $46 \mu \mathrm{S} \mathrm{cm}^{-1} \mathrm{~g}^{-1}$.

Na safra 1995/96, também houve diminuição nos valores da condutividade elétrica observada, decorrente da elevação do teor de água das sementes (Tabela 3). A cultivar Doko RC apresentou os menores valores; a Savana e a IAS-5 revelaram comportamento intermediário, e IAC-17, IAC-15 e IAC-31 Foscarin

Tabela 2. Condutividade elétrica observada $\mathrm{CE}_{0}$ e corrigida CE $\left(\mu \mathrm{Sm}^{-1} \mathrm{~g}^{-1}\right)$ de sementes de soja, de acordo com a cultivar e do teor de água inicial. Safra 1994/95 ${ }^{(1)}$.

\begin{tabular}{lcccccc}
\hline Cultivar & \multicolumn{7}{c}{ Teor de água (\%) } \\
\cline { 2 - 7 } & 7 & 9 & 11 & 13 & 15 & 17 \\
\hline \multirow{7}{*}{ Savana } & $106 \mathrm{dA}$ & $91 \mathrm{cB}$ & $80 \mathrm{cC}$ & $70 \mathrm{dD}$ & $62 \mathrm{cDE}$ & $60 \mathrm{dE}$ \\
Doko RC & $82 \mathrm{eA}$ & $65 \mathrm{~dB}$ & $58 \mathrm{dBC}$ & $53 \mathrm{eCD}$ & $49 \mathrm{dD}$ & $48 \mathrm{eD}$ \\
IAC-17 & $148 \mathrm{aA}$ & $125 \mathrm{aB}$ & $111 \mathrm{aC}$ & $107 \mathrm{aCD}$ & $100 \mathrm{aD}$ & $91 \mathrm{aE}$ \\
IAC-15 & $133 \mathrm{bA}$ & $117 \mathrm{aB}$ & $98 \mathrm{bC}$ & $88 \mathrm{bD}$ & $82 \mathrm{bDE}$ & $79 \mathrm{bE}$ \\
IAS-5 & $110 \mathrm{dA}$ & $93 \mathrm{cB}$ & $88 \mathrm{cB}$ & $76 \mathrm{cdC}$ & $70 \mathrm{cCD}$ & $67 \mathrm{cD}$ \\
IAC-31Fos & $123 \mathrm{cA}$ & $103 \mathrm{bB}$ & $86 \mathrm{cC}$ & $80 \mathrm{bcCD}$ & $80 \mathrm{bCD}$ & $79 \mathrm{bD}$ \\
\hline \multicolumn{7}{c}{$\mathrm{Condutividade}$ elétrica corrigida } \\
Savana & $72 \mathrm{cA}$ & $72 \mathrm{cA}$ & $71 \mathrm{cA}$ & $69 \mathrm{dA}$ & $67 \mathrm{cA}$ & $72 \mathrm{dA}$ \\
Doko RC & $56 \mathrm{dA}$ & $51 \mathrm{dAB}$ & $52 \mathrm{dAB}$ & $53 \mathrm{eAB}$ & $53 \mathrm{dAB}$ & $58 \mathrm{eB}$ \\
IAC-17 & $101 \mathrm{aA}$ & $98 \mathrm{aAB}$ & $98 \mathrm{aAB}$ & $106 \mathrm{aBC}$ & $110 \mathrm{aC}$ & $108 \mathrm{aC}$ \\
IAC-15 & $91 \mathrm{bA}$ & $92 \mathrm{aAB}$ & $87 \mathrm{bAB}$ & $87 \mathrm{bAB}$ & $89 \mathrm{bB}$ & $95 \mathrm{bB}$ \\
IAS-5 & $75 \mathrm{cA}$ & $73 \mathrm{cAB}$ & $78 \mathrm{cB}$ & $75 \mathrm{cdB}$ & $73 \mathrm{cB}$ & $84 \mathrm{cB}$ \\
IAC-31Fos & $83 \mathrm{bA}$ & $81 \mathrm{bB}$ & $76 \mathrm{cBC}$ & $79 \mathrm{cBC}$ & $82 \mathrm{bBC}$ & $95 \mathrm{bC}$ \\
\hline (1)Médias seguidas pela mesma letra, minúscula na coluna e maiúscula na \\
linha, não diferem entre si pelo teste de Tukey, a 5\% de probabilidade; os \\
coeficientes de variação relativos à condutividade elétrica observada e \\
corrigida foram de 7,90\% e 7,89\%, respectivamente.
\end{tabular}

Tabela 3. Condutividade elétrica observada $\mathrm{CE}_{\mathrm{o}}$ e corrigida $\mathrm{CE}\left(\mathrm{mS} \mathrm{cm}{ }^{-1} \mathrm{~g}^{-1}\right)$ de sementes de soja, de acordo com a cultivar e o teor de água inicial. Safra 1995/96(1).

\begin{tabular}{lcccccc}
\hline Cultivar & \multicolumn{5}{c}{ Teor de água (\%) } \\
\cline { 2 - 7 } & 7 & 9 & 11 & 13 & 15 & 17 \\
\hline \multirow{7}{*}{ Condutividade elétrica observada } \\
Davana & $115 \mathrm{aA}$ & $92 \mathrm{bB}$ & $79 \mathrm{bC}$ & $69 \mathrm{bD}$ & $65 \mathrm{cD}$ & $56 \mathrm{bE}$ \\
IAC-17 & $86 \mathrm{cA}$ & $68 \mathrm{cB}$ & $59 \mathrm{cC}$ & $50 \mathrm{cD}$ & $45 \mathrm{dDE}$ & $41 \mathrm{cE}$ \\
IAC-15 & $118 \mathrm{aA}$ & $103 \mathrm{aB}$ & $93 \mathrm{aC}$ & $85 \mathrm{aD}$ & $76 \mathrm{aE}$ & $65 \mathrm{aF}$ \\
IAS-5 & $119 \mathrm{aA}$ & $97 \mathrm{abB}$ & $89 \mathrm{aC}$ & $81 \mathrm{aD}$ & $76 \mathrm{aD}$ & $67 \mathrm{aE}$ \\
IAC-31Fos & $120 \mathrm{aA}$ & $93 \mathrm{bB}$ & $80 \mathrm{bC}$ & $72 \mathrm{bD}$ & $66 \mathrm{bcD}$ & $58 \mathrm{bE}$ \\
\hline \multicolumn{7}{c}{ Condutividade elétrica corrigida } \\
Savana & $78 \mathrm{abA}$ & $72 \mathrm{cB}$ & $70 \mathrm{bB}$ & $68 \mathrm{bB}$ & $70 \mathrm{bB}$ & $67 \mathrm{~dB}$ \\
Doko RC & $58 \mathrm{cA}$ & $53 \mathrm{dAB}$ & $52 \mathrm{cB}$ & $49 \mathrm{cB}$ & $50 \mathrm{cB}$ & $49 \mathrm{eB}$ \\
IAC-17 & $81 \mathrm{aA}$ & $81 \mathrm{aA}$ & $83 \mathrm{aA}$ & $84 \mathrm{aA}$ & $83 \mathrm{aA}$ & $78 \mathrm{abA}$ \\
IAC-15 & $81 \mathrm{aAB}$ & $76 \mathrm{abcB}$ & $78 \mathrm{aAB}$ & $80 \mathrm{aAB}$ & $82 \mathrm{aA}$ & $80 \mathrm{aAB}$ \\
IAS-5 & $73 \mathrm{bA}$ & $73 \mathrm{bcA}$ & $71 \mathrm{bA}$ & $71 \mathrm{bA}$ & $72 \mathrm{bA}$ & $69 \mathrm{cdA}$ \\
IAC-31Fos & $81 \mathrm{aA}$ & $78 \mathrm{abAB}$ & $78 \mathrm{aAB}$ & $81 \mathrm{aA}$ & $79 \mathrm{aA}$ & $73 \mathrm{bcB}$ \\
\hline
\end{tabular}

(1)Médias seguidas pela mesma letra, minúscula na coluna e maiúscula na linha, não diferem entre si pelo teste de Tukey, a 5\% de probabilidade; os coeficientes de variação relativos à condutividade elétrica observada e corrigida foram de $7,89 \%$ e $6,69 \%$, respectivamente. apresentaram os maiores valores, em todos os teores de água.

Variações na condutividade elétrica entre cultivares foram verificadas, também, por Panobianco \& Vieira (1996) e Vieira et al. (1996), com sementes de soja e feijão. Essas diferenças podem estar relacionadas a certas características da própria cultivar, como o teor de lignina no tegumento da semente (Alvarez et al., 1997), uma vez que existe uma estreita relação entre o teor de lignina no tegumento de sementes de soja e os resultados do teste de condutividade elétrica (Panobianco et al., 1999).

Os dados da condutividade elétrica corrigida (Tabela 3) confirmaram os obtidos na safra 1994/95 (Tabela 2). Verificou-se redução na amplitude de variação da condutividade elétrica entre os teores de água; a maior diferença apresentada foi de apenas $11 \mu \mathrm{S} \mathrm{cm}^{-1} \mathrm{~g}^{-1}$ (Savana). Comparando-se com os valores não-corrigidos de condutividade, em que ocorreram diferenças entre os teores de água de até $59 \mu \mathrm{S} \mathrm{cm}^{-1} \mathrm{~g}^{-1}$ (Savana e IAC-31 Foscarin), os resultados mostraram que a utilização da equação de correção proporcionou padronização dos resultados, eliminando o efeito do teor de água inicial, das sementes de soja, no teste de condutividade elétrica.

A condutividade elétrica diminuiu à medida que aumentou o teor de água das sementes; de maneira geral, a partir de $13 \%$ de água, os valores apresentaram padrão semelhante (Tabela 4). Nos dados corrigidos, a diferença máxima encontrada foi de $8 \mu \mathrm{S} \mathrm{cm}^{-1} \mathrm{~g}^{-1}$ (1995) e de $5 \mu \mathrm{S} \mathrm{cm} \mathrm{cm}^{-1} \mathrm{~g}^{-1}$ (1996), bem menor do que a revelada com os dados observados, ou seja: $45 \mu \mathrm{S} \mathrm{cm}^{-1} \mathrm{~g}^{-1}$ (1995) e $53 \mu \mathrm{S} \mathrm{cm}^{-1} \mathrm{~g}^{-1}$ (1996).

Tabela 4. Condutividade elétrica observada $\mathrm{CE}_{0}$ e corrigida $\mathrm{CE}\left(\mu \mathrm{S} \mathrm{cm}^{-1} \mathrm{~g}^{-1}\right)$, de acordo com o teor de água inicial das sementes de soja. Safras 1994/95 e 1995/96 $6^{(1)}$.

\begin{tabular}{cccccc}
\hline \multirow{2}{*}{$\begin{array}{c}\text { Teor de água } \\
(\%)\end{array}$} & \multicolumn{4}{c}{ Condutividade elétrica } \\
\cline { 2 - 3 } \cline { 2 - 5 } \cline { 5 - 6 } & Observada & Corrigida & & Observada & Corrigida \\
\hline 7 & $117 \mathrm{a}$ & $80 \mathrm{~b}$ & & $111 \mathrm{a}$ & $75 \mathrm{a}$ \\
9 & $99 \mathrm{~b}$ & $78 \mathrm{~b}$ & & $92 \mathrm{~b}$ & $72 \mathrm{~b}$ \\
11 & $87 \mathrm{c}$ & $77 \mathrm{~b}$ & & $81 \mathrm{c}$ & $72 \mathrm{~b}$ \\
13 & $79 \mathrm{~d}$ & $78 \mathrm{~b}$ & & $73 \mathrm{~d}$ & $72 \mathrm{~b}$ \\
15 & $73 \mathrm{e}$ & $79 \mathrm{~b}$ & & $67 \mathrm{e}$ & $73 \mathrm{~b}$ \\
17 & $72 \mathrm{e}$ & $85 \mathrm{a}$ & $58 \mathrm{f}$ & $70 \mathrm{c}$ \\
\hline $\mathrm{CV}(\%)$ & 7,90 & 7,89 & 6,56 & 6,69 \\
\hline
\end{tabular}

${ }^{(1)}$ Médias seguidas pela mesma letra, na coluna, não diferem entre si pelo teste de Tukey, a 5\% de probabilidade. 
Esses resultados permitem inferir que a equação de correção isolou o efeito do teor de água inicial das sementes com bastante precisão e enquadrou todos os lotes dentro de um padrão de vigor considerado como alto, segundo Vieira (1994).

Os dados obtidos no presente estudo indicaram a vantagem do uso da equação de correção, ao invés de se trabalhar somente com fatores de correção de teores de água predeterminados (Loeffler et al., 1988; Carvalho, 1994), uma vez que essa correção proporciona maior flexibilidade, principalmente em teores de água intermediários. Neste caso, haveria a necessidade de serem calculados fatores de correção para os teores, ao passo que com a equação de correção bastaria substituí-los, obtendo-se os valores corrigidos diretamente. Além disso, a equação permite utilizar os resultados da condutividade elétrica com maior segurança, uma vez que a comparação dos dados não é realizada entre lotes de sementes com diferentes padrões de teor de água, o que poderia levar a interpretações incorretas da real condição fisiológica das sementes. Os resultados após a correção não revelaram alteração na identificação do potencial fisiológico dos lotes.

\section{Conclusões}

1. O teor de água inicial das sementes de soja pode influenciar a interpretação dos resultados do teste de condutividade elétrica.

2. A equação de correção pode ser indicada para a correção dos resultados de condutividade elétrica de sementes de soja, independentemente do nível de vigor e da cultivar avaliada.

\section{Agradecimento}

À Fundação de Amparo à Pesquisa do Estado de São Paulo (Fapesp), pelo apoio financeiro.

\section{Referências}

ALVAREZ, P. J. C.; KRZYZANOWSKI, F. C.; MANDARINO, J. M. G.; FRANÇA NETO, J. B. Relationship between soybean seed coat lignin content and resistance to mechanical damage. Seed Science and Technology, Zurich, v. 25, n. 2, p. 209-214, 1997.
ASSOCIATION OF OFFICIAL SEED ANALYSTS (East Lasing, Estados Unidos). Seed vigor testing handbook. East Lasing, 1983. 93 p. (Contribution, 32).

BRASIL. Ministério da Agricultura. Regras para análise de sementes. Brasília: Secretaria Nacional de Defesa Agropecuária, 1992. 365 p.

CARVALHO, M. V. Determinação do fator de correção para condutividade elétrica em função do teor de água de sementes de soja [Glycine $\max$ (L.) Merrill]. Jaboticabal: Unesp, 1994. 36 p.

DELOUCHE, J. C.; BASKIN, C. C. Accelerated aging techniques for predicting the relative storability of seed lots. Seed Science and Technology, Zurich, v. 1, n. 2, p. 427-452, 1973.

DESWAL, D. P.; SHEORAN, I. S. A simple method for seed leakage measurement: applicable to single seeds of any size. Seed Science and Technology, Zurich, v. 21, n. 1, p. 179-185, 1993.

DIAS, D. C. F. S.; MARCOS FILHO, J. Testes de vigor baseados na permeabilidade das membranas celulares. I. Condutividade elétrica. Informativo Abrates, Londrina, v. 5, n. 1, p. 26-36, 1995.

GIVELBERG, A.; HOROWITZ, M.; POLJAKOFFMAYBER, A. Solute leakage from Solanum nigrum L. seeds exposed to high temperatures during imbibition. Journal of Experimental Botany, Oxford, v. 35, n. 161, p. 1754-1763, 1984.

HAMPTON, J. G.; JOHNSTONE, K. A.; EUAUMPON, V. Bulk conductivity test variables for mungbean, soybean and French bean seed lots. Seed Science and Technology, Zurich, v. 20, n. 3, p. 677-686, 1992.

HAMPTON, J. G.; LUNGWANGWA, A. L.; HILL, K. A. The bulk conductivity test for Lotus seed lots. Seed Science and Technology, Zurich, v. 22, n. 1, p. 177-180, 1994.

LOEFFLER, T. M.; TEKRONY, D. M.; EGLI, D. B. The bulk conductivity test as an indicator of soybean seed quality. Journal of Seed Technology, Lincoln, v. 12, n. 1, p. 37-53, 1988.

MARCOS FILHO, J. Testes de vigor: importância e utilização. In: KRZYZANOWSKI, F. C.; VIEIRA, R. D.; FRANÇA NETO, J. B. (Ed.). Vigor de sementes: conceitos e testes. Londrina: Abrates, 1999. cap. 1, p. 1-21. 
MURPHY, J. B.; NOLAND, T. L. Temperature effects on seed imbibition and leakage mediated by viscosity and membranes. Plant Physiology, Rockville, v. 69, n. 2, p. 428-431, 1982.

PANOBIANCO, M.; VIEIRA, R. D. Electrical conductivity of soybean soaked seeds. I. Effect of genotype. Pesquisa Agropecuária Brasileira, Brasília, v. 31, n. 9, p. 621-627, set. 1996.

PANOBIANCO, M.; VIEIRA, R. D.; KRZYZANOWSKI, F. C.; FRANÇA NETO, J. B. Electrical conductivity of soybean seed and correlation with seed coat lignin content. Seed Science and Technology, Zurich, v. 27, n. 3, p. 945-949, 1999.

TAO, J. K. Factors causing variations in the conductivity test for soybean seeds. Journal of Seed Technology, Lincoln, v. 3, n. 1, p. 10-18, 1978.

VIEIRA, R. D. Teste de condutividade elétrica. In: VIEIRA, R. D.; CARVALHO, N. M. (Ed.). Testes de vigor em sementes. Jaboticabal: Funep, 1994. p. 103-132.
VIEIRA, R. D.; KRZYZANOWSKI, F. C. Teste de condutividade elétrica. In: KRZYZANOWSKI, F. C.; VIEIRA, R. D.; FRANÇA NETO, J. B. (Ed.). Vigor de sementes: conceitos e testes. Londrina: Abrates, 1999. cap. 4, p. 1-26.

VIEIRA, R. D.; MINOHARA, L.; PANOBIANCO, M.; BERGAMASCHI, M. C. M.; MAURO, A. O. Comportamento de cultivares de soja quanto à qualidade fisiológica de sementes. Pesquisa Agropecuária Brasileira, Brasília, v. 33, n. 2, p. 123-130, fev. 1998.

VIEIRA, R. D.; PANOBIANCO, M.; LEMOS, L. B.; FORNASIERI FILHO, D. Efeito de genótipos de feijão e de soja sobre os resultados da condutividade elétrica de sementes. Revista Brasileira de Sementes, Brasília, v. 18, n. 2, p. 220-224, 1996.

WANG, Y. R.; HAMPTON, J. G.; HILL, M. J. Red clover vigour testing: effects of three test variables. Seed Science and Technology, Zurich, v. 22, n. 1, p. 99-105, 1994. 